\title{
Efeito da Massagem Clássica na Percepção Subjetiva de Dor, Edema, Amplitude Articular e Força Máxima Após Dor Muscular Tardia Induzida Pelo Exercício
}

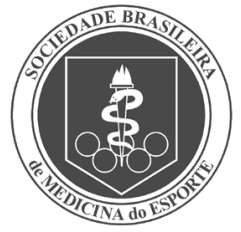

Artigo Original

\author{
Effect of Classical Massage on Subjective Perceived Soreness, Edema, \\ Range of Motion and Maximum Strength after Delayed Onset Muscle \\ Soreness Induced by Exercise
}

César Cavinato Cal Abad'1,2

Leonardo Takamitsu Ito'

Renato Barroso ${ }^{2}$

Carlos Ugrinowitsch ${ }^{2}$

Valmor Tricoli

1. Universidade Bandeirante de São Paulo (Uniban) - São Paulo - SP

2. Escola de Educação Física e Esporte da Universidade de São Paulo (EEFEUSP) - São Paulo - SP

Endereço para correspondência: César Cavinato Cal Abad Rua Maria Cândida, 1.813

V. Guilherme

02071-13 - São Paulo, SP

E-mail: c.cavinato@uol.com.br

\section{RESUMO}

O treino de força com cargas elevadas tem induzido indivíduos a apresentarem sintomas de dano muscular que incluem a dor muscular tardia. Na tentativa de diminuir sintomas e desconforto da DOMS, estratégias têm sido utilizadas, entre elas, a massagem. O objetivo do presente estudo foi verificar os efeitos da massagem clássica na percepção subjetiva de dor (DOMS), circunferência do braço (CIR), amplitude de movimento (ADM) e força máxima (1RM) após protocolo para indução de DOMS. Para isso, 18 adultos jovens saudáveis do gênero masculino foram divididos em três grupos ( $G 1$ = massagem; $G 2$ = protocolo; $G 3$ = protocolo + massagem) equalizados pelo teste de uma força máxima de flexão de cotovelo no banco Scott. O protocolo de indução de DOMS consistiu de 30 ações excêntricas musculares supramáximas (seis séries de cinco repetições a 110\% de 1RM). A massagem foi realizada no grupo G3 imediatamente após o protocolo durante seis minutos. As variáveis dependentes (DOMS, CIR, ADM) foram avaliadas 24, 48, 72 e 96 horas após o protocolo, enquanto a força máxima, apenas após 48 e 96 horas. Os resultados indicaram aumento na DOMS e diminuição na ADM e 1RM, similar aos de outros estudos que utilizaram protocolos semelhantes. No entanto, não houve diferenças entre os grupos G2 e G3 em nenhuma das variáveis analisadas. Pode-se concluir que com esse design experimental o protocolo utilizado foi eficaz para provocar as alterações nas variáveis analisadas e a massagem não causou nenhum benefício na recuperação das funções musculares nem na percepção subjetiva de dor.

Palavras-chave: treinamento, força, contração excêntrica.

\begin{abstract}
Heavy resistance training induces to symptoms of muscle damage which include delayed onset muscle soreness (DOMS). Some strategies (i.e. massage) have been used to attenuate these symptoms and to reduce discomfort associated with DOMS. This study aimed to investigate the effects of classical massage on DOMS perception, limb girth (CIR), range of motion (ADM) and maximum strength performance (1-RM) after a muscle damage protocol. Eighteen males were divided into three groups (G1= massage-only, G2= protocol-only, G3= protocol + massage) according to their 1-RM values. DOMSinducing protocol consisted of 30 supramaximal eccentric contractions (6 sets of 5 repetitions at 110\% 1RM). Immediately after the protocol, classical massage was performed in G3 for 6 minutes. DOMS, CIR, ADM were assessed 24, 48, 72 and 96 hours after the muscle damage protocol, and maximum strength was evaluated only after 48 and 96 hours. Results showed increase in DOMS and reduction in ADM and 1-RM values, in agreement with other studies using similar procedures. However, there was no difference between $\mathrm{G} 2$ and $\mathrm{G} 3$ in any of the assessed variables. It can be concluded that the muscle damage protocol used in this study was effective in causing alteration in the dependent variables observed. However, classical massage was not effective to minimize the symptoms associated to exercise induced muscle damage.
\end{abstract}

Keywords: training, strength, eccentric contraction. 


\section{INTRODUÇÃO}

Após a prática de exercício extenuante de força, o corpo humano reage com manifestações de desconforto, diminuição da amplitude de movimento e edema muscular. Essas manifestações limitam a realização das atividades diárias e da prática esportiva e estão associadas ao dano muscular.

O dano muscular pode ser medido de forma direta por amostras musculares ou pela técnica de ressonância magnética e de forma indireta pela análise das concentrações de enzimas plasmáticas, proteínas musculares e mioglobina no sangue, amplitude de movimento, edema muscular, diminuição da força máxima e dor muscular tardia (DOMS) $)^{(1)}$. Pela dificuldade de acessibilidade da técnica invasiva ou pelo alto dispêndio econômico, na prática, os métodos mais utilizados para estimativa do dano muscular têm sido a avaliação da força máxima (1RM), as medidas de circunferência dos segmentos corporais para averiguação de edema e principalmente a percepção subjetiva de dor muscular tardia(2).

O dano muscular é causado por lesões na fibra que afetam as estruturas celulares (linha Z, sarcolema, túbulos T, miofibrilas e proteínas estruturais) e tecido conectivo e provocam inflamação local pela migração de leucócitos. Essa inflamação induz a liberação de histaminas, prostaglandinas, cininas e potássio, que ativam nociceptores profundos (terminações nervosas de dor), causando sensação de dor e desconforto ${ }^{(3-5)}$.

A DOMS é caracterizada pela dor que afeta a musculatura esquelética exercitada após a realização de movimentos não rotineiros ou treinamentos de alta intensidade com grande conteúdo excêntrico e pode afetar indivíduos sedentários ou treinados. A DOMS surge principalmente após um período de 24 horas, atinge seu pico entre 48 e 72 horas e diminui entre cinco e sete dias. Também prejudica o desempenho físico e retarda a recuperação(6).

Na tentativa de prevenir ou amenizar seus sintomas e efeitos, várias estratégias têm sido investigadas. Entre elas encontram-se o alongamento, a crioterapia, a estimulação elétrica, o ultrassom, as drogas antiinflamatórias e a hidroterapia ${ }^{(7)}$. Outro recurso aplicado para amenizar as dores e otimizar a recuperação é a massagem. Embora não seja recomendada ${ }^{(8,9)}$ para proteção muscular, aumento de desempenho e aceleração de recuperação, a massagem parece reduzir significativamente a percepção subjetiva de dor e o edema muscular ${ }^{(2,7)}$.

Porém, os efeitos da massagem ainda são controversos. Segundo Ernst $^{(10)}$ e Moraska ${ }^{(11)}$, esse fato se dá devido às limitações dos estudos que abordaram o problema. Os diferentes protocolos de indução de DOMS, a quantidade de massa muscular envolvida, as diferentes técnicas de massagem empregadas (clássica, esportiva), os períodos de aplicação, a duração, a intensidade e a frequência de aplicação das massagens são fatores que dificultam as conclusões sobre sua eficiência.

Assim, o objetivo do presente estudo foi verificar os efeitos da massagem clássica na percepção subjetiva de DOMS e nos marcadores indiretos de dano muscular (circunferência, amplitude de movimento e o desempenho de força máxima)

\section{MÉTODOS}

O estudo foi aprovado pelo comitê de ética local. Os sujeitos foram informados dos procedimentos, benefícios e riscos do estudo e assinaram termo de consentimento antes da participação.

A amostra foi constituída de 18 sujeitos fisicamente ativos, do gênero masculino, com idade entre 18 e 30 anos. Os sujeitos foram divididos em três grupos com seis em cada; a divisão dos grupos foi realizada após o ranqueamento dos indivíduos com base em seus valores de 1RM para flexão de cotovelos.
O grupo I (G1) não realizou o protocolo de DOMS e só recebeu a massagem, o grupo ॥ (G2) realizou o protocolo de DOMS e não recebeu massagem e o grupo III (G3) realizou o protocolo de DOMS e recebeu a massagem.

Para participar do estudo, os indivíduos não deveriam apresentar lesões articulares, musculares e/ou ligamentares no cotovelo e/ou punho, nem fazer uso de medicamento e/ou suplemento alimentar 30 dias antes do início e até o final do estudo. Além disso, não poderiam praticar treinamento de força para membros superiores seis meses antes do início do protocolo experimental. Todos os sujeitos foram orientados a manter o mesmo padrão de vida e de atividades durante todo o período de experimento.

Antes de se iniciar a coleta de dados, os voluntários realizaram visitas ao laboratório para familiarização com os equipamentos e os procedimentos utilizados.

Os testes e os procedimentos utilizados para obtenção das medidas encontram-se detalhados a seguir.

\section{Teste de força dinâmica máxima (1RM)}

Para mensuração da força dinâmica máxima, foi realizado o teste de uma repetição máxima (1RM) de flexão de cotovelo unilateral. 0 movimento foi realizado com auxílio de um banco Scott, onde os sujeitos foram posicionados sentados com o braço apoiado no banco na posição supina, mantendo o tronco estável durante todo o teste. Esse teste foi realizado antes (Pré), imediatamente depois (Pós), 48 e 96 horas após o término do protocolo de indução da DOMS.

Antes da realização do teste, para efeito de aquecimento geral, o indivíduo realizou caminhada a $7 \mathrm{~km} / \mathrm{h}$ durante cinco minutos e antes do aquecimento localizado foi feita uma sessão de cinco minutos de exercícios de alongamento passivo para os membros superiores. 0 aquecimento específico constou de uma série de 10 repetições de flexões do cotovelo com uma barra de $3 \mathrm{~kg}$ seguida de uma série de cinco repetições com 50\% da carga estimada para 1RM e uma série de três repetições com 70\% da carga estimada para 1RM. Foi dado um intervalo de dois minutos entre as séries de aquecimento e para o início do teste os sujeitos descansaram três minutos.

A determinação da 1RM foi realizada no máximo em cinco tentativas com intervalo de três minutos entre elas. A execução técnica do movimento foi avaliada pelo pesquisador responsável. A tentativa foi considerada válida quando os sujeitos executaram um ciclo completo de movimento. O ciclo iniciava-se com a flexão do cotovelo a 30, passando pela extensão completa, retornando à posição inicial.

Para reduzir o erro no teste de 1RM foram adotadas as seguintes estratégias: 1) instruções padronizadas foram oferecidas antes do teste, de modo que o sujeito estivesse ciente de toda a rotina que envolvia a coleta de dados; 2) sujeitos foram familiarizados com a técnica de execução do exercício; 3) manutenção da posição do tronco e do segmento corporal no momento da execução dos movimentos; 4) os testes foram realizados nos mesmos horários; 5) foram utilizadas uma barra e anilhas com precisão de 0,5kg.

A avaliação dos marcadores indiretos de dano e DOMS foi realizada antes (Pré), imediatamente depois (Pós), 24, 48, 72 e 96 horas subsequentes ao término do protocolo de indução da DOMS.

\section{Amplitude de movimento (ADM)}

A ADM foi calculada pela diferença entre o ângulo do cotovelo relaxado e o de máxima flexão. A medida da amplitude de movimento da articulação do cotovelo foi realizada com um goniômetro de plástico transparente com duas réguas para mensuração de amplitude articular, sistema de transferidor de $0^{\circ}$ a $360^{\circ}$ e medidas aproximadas 
de 0,20cm x 0,05cm (Carci, São Paulo, Brasil). Os pontos anatômicos de referência para o posicionamento do goniômetro foram a inserção do deltoide, o epicôndilo lateral do úmero e o ponto médio entre os processos estiloides ulnar e radial. Esses pontos foram marcados com tinta semipermanente para a manutenção dos mesmos durante todo o período do estudo. O ângulo do cotovelo relaxado foi medido com os sujeitos em pé e com os braços relaxados ao lado do corpo. O ângulo de flexão foi avaliado ao final da flexão voluntária máxima depois de pedido ao sujeito que tocasse o ombro ipsilateral com a palma da mão.

\section{Circunferência do braço (CIR)}

A CIR foi medida com uma trena metálica (American Medical do Brasil, São Paulo, Brasil) na região de maior perímetro. Essa medida foi realizada com o sujeito em pé e com o braço ao longo do corpo em posição neutra ${ }^{(12)}$.

\section{Dor muscular tardia (DOMS)}

Para mensurar a DOMS, foi utilizada uma escala de percepção subjetiva de dor. Os sujeitos receberam uma folha com uma linha de $10 \mathrm{~cm}$ de comprimento, que representava uma escala subjetiva de dor onde uma extremidade $(0 \mathrm{~cm})$ se referia a "normal" e a outra $(10 \mathrm{~cm})$ a "muito dolorido"(2). O sujeito deveria indicar na linha um ponto que melhor representasse a sensação de dor sentida por ele naquele momento. A distância em centímetros da extremidade zero $(0 \mathrm{~cm})$ até o ponto indicado pelo sujeito foi considerada como a medida de dor.

\section{Protocolo para a indução da DOMS}

O exercício escolhido para induzir a DOMS foi a flexão de cotovelo no banco Scott. Para minimizar a interferência da dor na realização das atividades cotidianas dos indivíduos, o protocolo de exercício foi realizado com o braço não dominante.

O protocolo de DOMS foi realizado três minutos após o teste de força dinâmica máxima e constou de 30 ações excêntricas supramáximas (110\% de 1RM) divididas em cinco séries de seis repetições e intervalos de três minutos entre as séries. A velocidade de execução das repetições foi controlada através de um metrônomo digital (Seiko DM-20), sendo três segundos por fase do movimento. A fase excêntrica foi realizada voluntariamente pelo indivíduo e a fase concêntrica foi auxiliada pelo pesquisador. A amplitude de movimento foi semelhante à utilizada no teste de 1RM e foi constantemente controlada.

Durante o protocolo, cada repetição em que houve falha na velocidade de execução ou na amplitude de movimento foi desconsiderada e o indivíduo as realizou depois da última série. Caso houvesse duas falhas seguidas, a série era interrompida e não válida, tendo que ser completada ao final do protocolo.

\section{Técnica de massagem}

A técnica de massagem foi a denominada clássica, a qual foi realizada imediatamente após o protocolo de indução da DOMS. Os indivíduos do G1 e G3 receberam seis minutos de massagem na musculatura flexora do cotovelo. O tempo total de massagem foi dividido em: 1) deslizamento superficial (30 segundos); 2) deslizamento profundo (30 segundos); 3) amassamento (um minuto); 4) fricções com os polegares (um minuto); 5) fricções com os quatro dedos (um minuto); 6) percussões com as mãos abertas e alternadas (30 segundos); 7) percussões com as mãos abertas e simultâneas (30 segundos); 8) tapotagem (30 segundos); e 9) deslizamento superficial (30 segundos).
A massagem foi aplicada por um mesmo terapeuta, especialista em massagem clássica, com instruções de manter o mais idêntico possível todos os procedimentos. Durante a massagem foi aplicada vaselina líquida neutra e sem princípios ativos que pudessem interferir na DOMS ou na massagem da musculatura.

\section{Análise estatística}

Para estatística descritiva calculou-se a mediana e os valores máximos e mínimos foram apresentados das variáveis antropométricas.

Para comparações de médias, como os dados não apresentaram distribuição normal, foram utilizados os testes não paramétricos de Wilcoxon para as amostras pareadas (intragrupos) e de Mann-Whitney para amostras independentes (entre grupos). Para realização de todos os cálculos utilizou-se o programa Instat e adotou-se nível de significância de $p \leq 0,05$.

\section{RESULTADOS}

As características antropométricas dos sujeitos de cada grupo são apresentadas na tabela 1.

Tabela 1. Características antropométricas de cada grupo (mediana, máximo e mínimo)

\begin{tabular}{c|c|c|c|c|c|c|c|c|c}
\hline \multirow{2}{*}{ GRUPO } & \multicolumn{2}{|c|}{ Massa corporal (kg) } & \multicolumn{3}{|c|}{ Estatura (m) } & \multicolumn{3}{c}{ IMC } \\
\cline { 2 - 9 } & MED & MÁX & MÍN & MED & MÁx & MÍN & MED & MÁx & MíN \\
\hline G1 & 63,50 & 94,60 & 58,50 & 1,81 & 1,87 & 1,66 & 20,85 & 27,79 & 18,63 \\
\hline G2 & 70,75 & 79,70 & 59,80 & 1,79 & 1,87 & 1,70 & 22,14 & 24,82 & 20,74 \\
\hline G3 & 76,10 & 122,90 & 63,40 & 1,74 & 1,87 & 1,70 & 24,91 & 35,15 & 20,94 \\
\hline
\end{tabular}

Os resultados de DOMS, CIR, ADM e 1RM para cada grupo são apresentados nas figuras 1 a 4. Para a variável DOMS foi encontrado apenas efeito de tempo nos grupos G2 (Pré x 24h; Pré x 48h; Pré x 72h) e G3 (Pré x 24h; Pré x 48h; Pré x 72h; Pós x 48h; 48h x 96h) (figura 1). O mesmo ocorreu para a ADM no G2 (Pré x 24h) e no G3 (Pré x Pós; Pré x 24h) (figura 2); e para o teste de força máxima nos grupos G2 (Pós x 96h) e G3 (Pré x Pós; Pré x 48h) (figura 3). Não foi encontrada nenhuma diferença significante nos valores de CIR (figura 4). Diferenças significantes intergrupos foram encontradas somente em ADM (Pós G2 x Pós G3 e 96h G2x 96h G3) (figura 2).

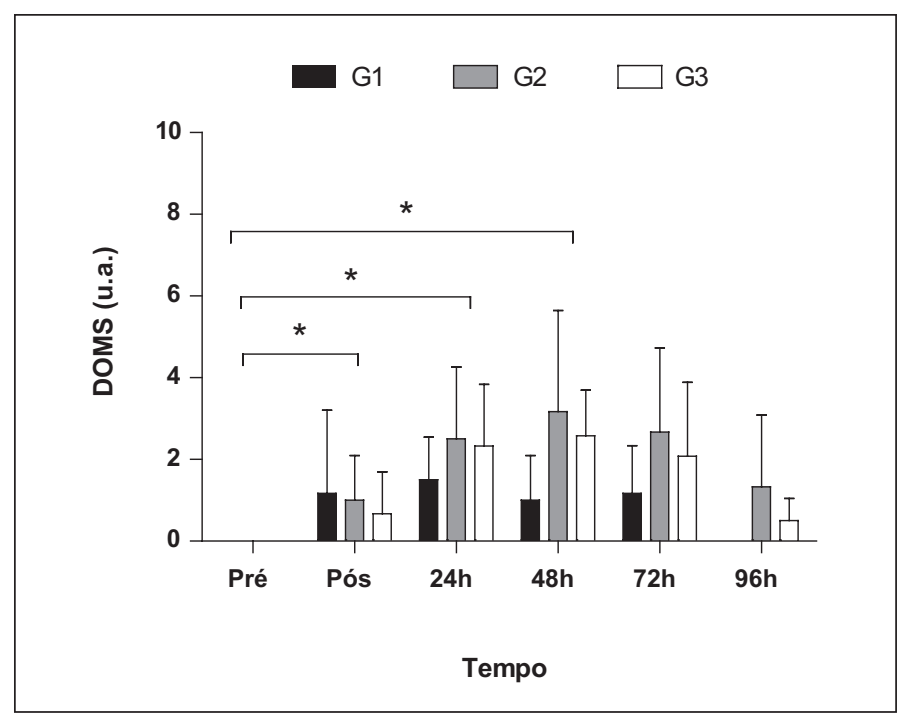

* $p<0,05$ em relação à condição Pré para G2 (protocolo) e G3 (protocolo e massagem).

Figura 1. Percepção subjetiva de dor (DOMS) nos diferentes tempos em cada grupo 


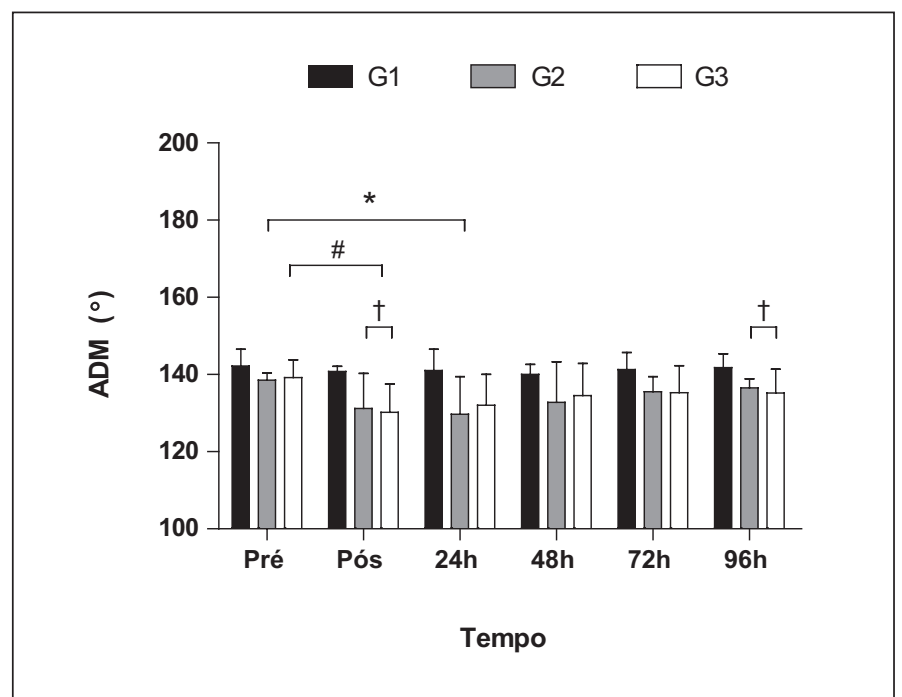

${ }^{*} \mathrm{p}<0,05$ em relação à condição Pré para G2 (protocolo) e G3 (protocolo e massagem)

" $\mathrm{p}<0,05$ Pré vs. Pós para o grupo G3 (protocolo e massagem)

${ }^{\dagger} p<0,05$ G2 vs. G3.

Figura 2. Amplitude de movimento (ADM) nos diferentes tempos em cada grupo

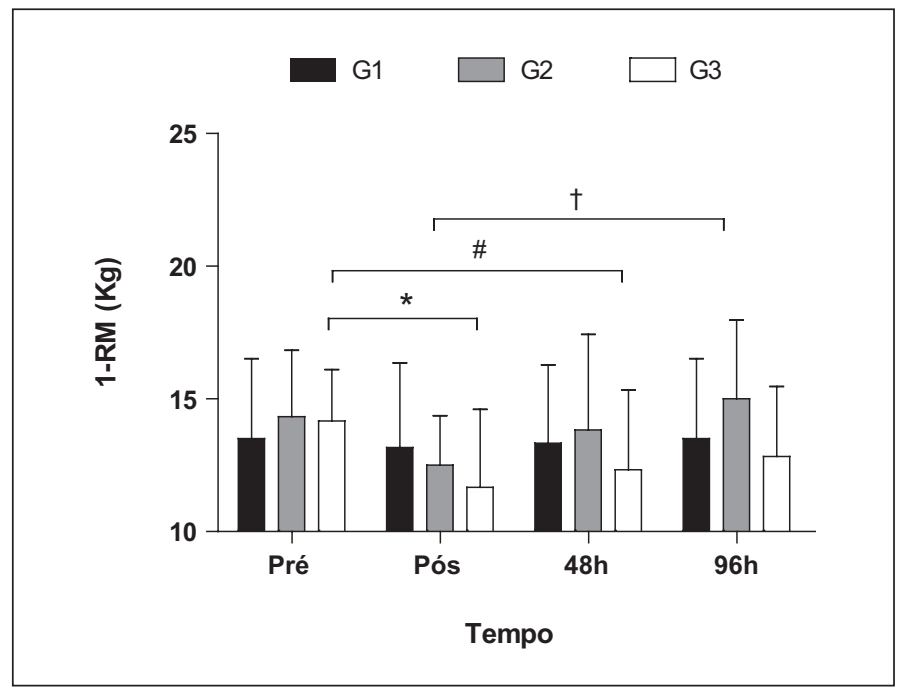

* $\mathrm{p}<0,05$ Pré vs Pós para o grupo G3 (protocolo e massagem)

"p $<<0,05$ Pré vs. 48 horas para o grupo G3 (protocolo e massagem)

${ }^{\dagger} p<0,05$ Pós vs. 96 horas para o grupo G2 (protocolo).

Figura 3. Força máxima (1RM) nos diferentes tempos em cada grupo

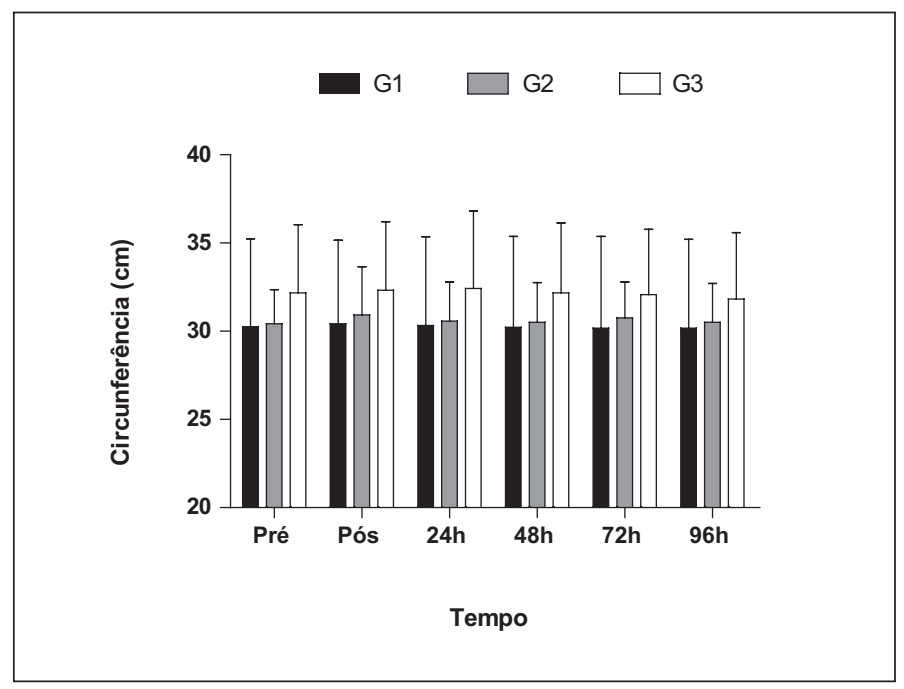

Figura 4. Circunferência de braço nos diferentes tempos em cada grupo

\section{DISCUSSÃO}

O objetivo deste estudo foi observar o efeito da massagem clássica na percepção subjetiva de dor e nos marcadores indiretos de dano muscular (CIR, força máxima, ADM). Apesar de pouco estudada e seus efeitos não serem claros, a massagem é um estratégia bastante utilizada por técnicos de modalidades esportivas no intuito de acelerar a recuperação dos atletas. Dessa maneira, era esperado que a utilização dessa técnica pudesse auxiliar na recuperação das funções musculares avaliadas através da amplitude de movimento e força muscular, além da diminuição da dor e edema. O grupo 3 (exercício + massagem) e o grupo 2 (exercício) não apresentaram diferenças significantes entre si durante o período avaliado no presente estudo, indicando que a massagem não teve efeito na percepção subjetiva de dor nem nos marcadores indiretos de dano muscular.

A DOMS é caracterizada pela sensação de desconforto e dor na musculatura esquelética que ocorre algumas horas após a prática de uma atividade física à qual os indivíduos não estejam acostumados. Essa dor não se manifesta até aproximadamente oito horas após o exercício, mas aumenta de intensidade durante as primeiras 24 horas e alcança seu pico entre 48 e 72 horas e cinco a sete dias retorna aos valores iniciais ${ }^{(7)}$. Esse mesmo padrão de evolução da DOMS foi observado no presente estudo. O pico de dor aconteceu 48 horas após o protocolo para induzir DOMS em ambos os grupos que realizaram o protocolo (G2 e G3) e 96 horas pós-exercício já havia retornado ao mesmo nível de antes da realização do protocolo. É interessante notar que a massagem isolada também provocou o aumento da dor até 24 horas pós exercício. Porém, a massagem realizada após o protocolo não exacerbou a dor percebida pelos indivíduos.

Zainuddin et al. (2) realizaram um estudo semelhante ao nosso com a realização de 60 ações excêntricas máximas no dinamômetro isocinético $\left(90^{\circ} \cdot 5^{-1}\right)$ e massagem 30 minutos após as ações excêntricas. Ao contrário do presente experimento, esses autores observaram diminuição na dor e nos marcadores indiretos de dano muscular observados por um período de 14 dias. É possível que a característica do exercício e a carga aplicada durante as ações excêntricas tenham provocado essas diferenças. No estudo de Zainuddin et al.(2) as ações excêntricas foram máximas e realizadas no dinamômetro isocinético, enquanto que nosso estudo utilizou ações isoinerciais com carga de 110\% de 1RM máximo. Durante o teste de uma repetição máxima (1RM) a carga máxima que o sujeito consegue superar é determinada pela sua capacidade de geração de tensão durante a ação concêntrica. Como as ações excêntricas têm maior capacidade de geração de tensão do que as concêntricas, então a carga utilizada durante nosso protocolo foi de natureza submáxima para as ações excêntricas. Esse fato pode ter influenciado a ocorrência de dano muscular, visto que a força desenvolvida durante as ações musculares afeta o grau de dano muscular induzido. Assim, é possível que a massagem afete a percepção subjetiva de dor somente quando o grau de dano ocorrido é elevado.

Essa hipótese se confirma quando são analisados os dados de Howatson e Van Someren ${ }^{(13)}$. Nesse estudo, os autores utilizaram exercícios isoinerciais com cargas submáximas (70\% de 1RM) e aplicaram massagem com a utilização de gelo na tentativa de diminuir a resposta dos marcadores indiretos de dano muscular. Com exceção da atenuação da resposta da CK (creatina quinase - enzima intramuscular), a massagem não apresentou benefícios em nenhuma das variáveis analisadas (DOMS, CIR, ADM, 1RM). No nosso estudo, a concentração plasmática de CK não foi analisada, mas as respostas das outras variáveis apresentaram comportamento semelhante ao do estudo de Howatson e Van Somersen ${ }^{(13)}$. 
Além de o efeito da massagem na DOMS não estar bem estabelecido e haver controvérsia sobre o assunto, não existem estudos reportando qual o melhor momento para a aplicação da massagem. No nosso estudo, a massagem foi aplicada imediatamente após a realização do protocolo, enquanto que no de Zainuddin et al.(2), após 30 minutos. Esses autores encontraram efeito benéfico da massagem na DOMS (menor percepção dos indivíduos) e menor elevação dos marcadores de dano. Dessa forma, é possível que a aplicação mais tardia da massagem possa provocar benefícios mais pronunciados.

Outros estudos observaram a resposta muscular à aplicação repetitiva da massagem após um protocolo para induzir dano. Butterfield et al. ${ }^{(14)}$ observaram melhora nas respostas dos marcadores de dano em ratos após a realização de um protocolo para induzi-lo com a aplicação de massagem. Esses resultados são semelhantes aos encontrados por Tiidus e Showmaker ${ }^{(15)}$.
Contudo, foi observado que os padrões temporais do desenvolvimento dos marcadores de dano e da dor apresentam comportamentos distintos. Dessa maneira, parece que a dor não está diretamente relacionada com o dano muscular. Nosaka et al. ${ }^{(16)}$ sugeriram que a dor seja causada por dano no tecido conectivo presente na estrutura muscular (endomísio, epimísio e perimísio) e não na fibra muscular.

No presente estudo, ao contrário do observado em outros, a massagem não promoveu nenhum benefício em nenhuma das variáveis analisadas. Contudo, ressaltamos que o número de sujeitos no presente era pequeno, o que nos proporciona baixo poder estatístico. Sendo assim, o presente estudo não dá suporte à prática de treinadores e praticantes de atividade física utilizarem a massagem como forma de acelerar a recuperação das funções musculares e diminuição de dor.

Todos os autores declararam não haver qualquer potencial conflito de interesses referente a este artigo.

\section{REFERÊNCIAS BIBLIOGRÁFICAS}

1. McHugh MP. Recent advances in the understanding of the repeated bout effect: the protective effect against muscle damage from a single bout of eccentric exercise. Scand J Med Sci Sports 2003;13:88-97.

2. Zainuddin Z, Newton M, Sacco P, Nosaka K. Effects of massage on delayed-onset muscle soreness, swelling, and recovery of muscle function. J Athl Train 2005;40:174-80.

3. Friden J, Lieber RL. Structural and mechanical basis of exercise induced muscle injury. Med Sci Sports Exerc 1992;24:521-30.

4. Gibala MJ, MacDougall JD, Tarnopolsky MA, StauberWT, Elorriaga A. Changes in human skeletal muscle ultrastruture and force production after acute resistance exercise. J Appl Physiol 1995;78:702-8.

5. Armstrong RB, Warren GL, Warren JA. Mechanisms of exercise-induced muscle fibre injury. Sports Med 1991;12:184-207.

6. Clarkson PM, Hubal MJ. Exercise-induced muscle damage in humans. Am J Phys Med Rehabil 2002;81(11 Suppl):S52-69

7. Cheung K, Hume P, Maxwell L. Delayed onset muscle soreness: treatment strategies and performance factors. Sports Med 2003;33:145-64.

8. Barnett A. Using recovery modalities between training sessions in elite athletes: does it help? Sports Med 2006:36:781-96.
9. Howatson G, van Someren KA. The prevention and treatment of exercise-induced muscle damage. Sports Med 2008;38:483-503.

10. Ernst E. Does post-exercise massage treatment reduce delayed onset muscle soreness? A systematic review. Br J Sports Med 1998;32:212-4.

11. Moraska A. Sports massage. A comprehensive review. J Sports Med Phys Fitness 2005;45:370-80.

12. Evans RK, Knight KL, Draper DO, Parcell AC. Effects of warm-up before eccentric exercise on indirect markers of muscle damage. Med Sci Sports Exerc 2002;34:1892-9.

13. Howatson G, Van Someren KA. Ice massage. Effects on exercise-induced muscle damage. J Sports Med Phys Fitness 2003;43:500-5

14. Butterfield TA, Zhao Y, Agarwal S, Haq F, Best TM. Cyclic compressive loading facilitates recovery after eccentric exercise. Med Sci Sports Exerc 2008;40:1289-96.

15. Tiidus PM, Shoemaker JK. Effleurage massage, muscle blood flow and long-term post-exercise strength recovery. Int J Sports Med 1995;16:478-83.

16. Nosaka K, Newton M, Sacco P. Delayed-onset muscle soreness does not reflect the magnitude of eccentric exercise-induced muscle damage. Scand J Med Sci Sports 2002;12:337-46. 